\title{
Expression of GOLPH2 is associated with the progression of and poor prognosis in gastric cancer
}

\author{
GUANGLIN LIU $^{1}$, YAN ZHANG ${ }^{2}$, FEN HE $^{1}$, JIANFENG LI $^{1}$, XUAN WEI $^{1}$, YANG LI ${ }^{1}$, \\ XIAOWEN LIAO ${ }^{1}$, JIANCONG SUN ${ }^{1}$, WEI YI ${ }^{1}$ and DAOLI NIU ${ }^{1}$ \\ ${ }^{1}$ Department of Radiation Oncology, The First Affiliated Hospital of Guangzhou Medical University; \\ ${ }^{2}$ Department of Medical Oncology, The Sixth Affiliated Hospital of \\ Sun Yat-Sen University, Guangzhou, Guangdong, P.R. China
}

Received May 19, 2014; Accepted July 10, 2014

DOI: $10.3892 / o r .2014 .3404$

\begin{abstract}
Golgi phosphoprotein 2 (GOLPH2) has been associated with the development and progression of various human cancers. The aims of this study were to investigate the relationship between GOLPH2 and gastric cancer (GC) progression and explore the clinical significance of GOLPH2 in GC. GOLPH2 expression was examined in four pairs of primary GC tissues and the adjacent non-cancerous tissues from the same patients, using immunohistochemistry (IHC), quantitative PCR and western blotting. Furthermore, GOLPH2 protein expression was analyzed in 10 normal gastric tissues and 385 clinicopathologically characterized cases of GC by IHC. Statistical analyses were performed to determine the prognostic and diagnostic associations. GOLPH2 mRNA and protein expression were both markedly upregulated in GC tissues, compared with the paired adjacent non-cancerous tissues. The Chi-square test and Spearman analysis revealed a significant correlation between GOLPH2 expression and clinical stage, T classification, lymph node metastasis, metastasis and venous invasion. Patients with a higher GOLPH2 expression had a shorter overall survival (OS), compared to patients with lower GOLPH2 expression. Notably, our results suggested that GOLPH2 is associated with the development and progression of GC. Therefore, additional studies focusing on the potential of GOLPH2 as a novel therapeutic target in GC are required.
\end{abstract}

\section{Introduction}

Gastric cancer (GC) is the third most common cancer and the second leading cause of cancer-related mortalities worldwide (1). Over one of third all GC cases occur in China (2). Currently, surgery remains the first choice of treatment for GC.

Correspondence to: Professor Daoli Niu, Department of Radiation Oncology, The First Affiliated Hospital of Guangzhou Medical University, 151 Yanjiang Road, Guangzhou, Guangdong 510120, P.R China

E-mail: daoliniu@163.com

Key words: Golgi phosphoprotein 2, gastric cancer, prognosis, biomarker
However, the 5-year survival rate for patients with all stages remains $\sim 20 \%$, even after the improved prognosis of patients with GC resulting from the development of adjuvant therapy (3). Findings of previous studies have demonstrated that multiple genetic alterations are associated with the development and progression of GC, including alterations to tumor-suppressor genes, oncogenes, apoptosis-related genes, cell-cycle regulators and cell growth factors. Numerous aberrantly expressed genes have been associated with poor prognosis in GC, including Bmi-1 (4), cyclooxygenase-2 (COX-2) (5), bone morphogenetic-7 (BMP-7) (6), human epidermal growth factor receptor 2 (HER2) (7) and sphingosine kinase 1 (SPHK1) (8). However, no series of biomarkers is capable of personalizing the adjuvant chemotherapy, even though adjuvant chemotherapy has shown an increased survival after surgery. To improve prognosis and provide novel chemotherapeutic targets, it is important to characterize the molecular mechanisms underlying GC, which may also help to identify more useful prognostic biomarkers.

Golgi phosphoprotein 2 (GOLPH2) is a 73-kDa Golgi membrane glycoprotein that is normally located within the cis-Golgi complex. The expression of GOLPH2 mRNA was first described in the liver tissue of a patient with syncytial giant cell hepatitis (9). GOLPH2 is constitutively expressed by cells of the epithelial lineage, including prostatic epithelial cells, colonic enterocytes, proximal and distal epithelial cells, bronchial epithelial cells and hepatic biliary epithelial cells (9). The upregulation of GOLPH2 has been observed in hepatocellular carcinoma (HCC) (10), prostate cancer (11), renal cell cancer (12), seminoma (13) and lung cancer (14). Moreover, increased levels of serum GOLPH2 (sGOLPH2) were detected in blood specimens from patients with 14 different types of cancer, including GC (10).

Although the expression of GOLPH2 has been studied in several types of cancer, the association between GOLPH2 and $\mathrm{GC}$ remains unknown. In this study, the expression of GOLPH2 was analyzed in GC tissues. Moreover, to the best of our knowledge, this study has shown for the first time that the expression of GOLPH 2 correlated significantly with clinical stage, $\mathrm{T}$ classification, lymph node metastasis, metastasis and venous invasion in GC. Results of this study also showed that GOLPH2 is a potentially useful independent biomarker for prognosis in GC patients. 


\section{Materials and methods}

Patient information and tissue specimens. This study was conducted on paraffin-embedded samples from a total of 385 GC patients, who were histopathologically and clinically diagnosed at The First Affiliated Hospital of Guangzhou Medical University from January, 2008 to December, 2009. Ten normal human gastric tissues were also obtained from partial gastrectomy of patients with gastric ulcers. Four specimens of GC tissues and the matched adjacent non-cancerous gastric tissues from the same patients were also obtained and immediately frozen at freezing tube and stored in liquid nitrogen until further use.

In order to use these clinical materials for research purposes, patients provided prior consent and approval for the study was obtained from the Institutional Research Ethics Committee of the First Affiliated Hospital of Guangzhou Medical University (Guangdong, China). Clinicopathological classification and staging were determined according to the criteria proposed by the American Joint Committee on Cancer and International Union Against Cancer criteria. The clinicopathological characteristics of the patients are shown in Table I.

RNA extraction and quantitative PCR $(q P C R)$. Total RNA was extracted from the primary tumor materials using TRIzol reagent (Invitrogen, Carlsbad, CA, USA) according to the manufacturer's instructions. The RNA was pretreated with RNase-free DNase (Promega, Madison, WI, USA) and $2 \mu \mathrm{g}$ of RNA from each sample was used for random hexamerprimed cDNA synthesis. For the PCR-mediated amplification of GOLPH2 cDNA, an initial amplification using GOLPH2specific primers was performed with a denaturation step at $95^{\circ} \mathrm{C}$ for $10 \mathrm{~min}$, followed by 28 cycles of denaturation at $95^{\circ} \mathrm{C}$ for $60 \mathrm{sec}$, primer annealing at $58^{\circ} \mathrm{C}$ for $30 \mathrm{sec}$ and extension at $72^{\circ} \mathrm{C}$ for $30 \mathrm{sec}$, followed by a final extension at $72^{\circ} \mathrm{C}$ for 5 min. The cDNA samples were stored at $4^{\circ} \mathrm{C}$.

qPCR was employed to quantify the expression of GOLPH2 mRNA in each primary GC tissue, relative to the paired normal gastric tissues obtained from the same patient. qPCR amplification was performed, in triplicate for each reaction, using the SYBR-Green kit (Invitrogen , Carlsbad, CA, USA). The qPCR primers for GOLPH2 and glyceraldehyde3-phosphate dehydrogenase (GAPDH) were designed using Primer Express software version 2.0 software (Applied Biosystems). The primer sequences used were: GOLPH2, 5'-ATCCGAGTGCTGCAAGACCAGTTA-3' (forward), 5'-TCTGATTGATGCACTGGCTCAGGT-3' (reverse); GAPDH, 5'-GACTCATGACCACAGTCCATGC-3' (forward), 5'-AGAGGCAGGGATGATGTTCTG-3' (reverse). The GOLPH2 mRNA expression data were initially normalized to the geometric mean of the housekeeping gene GAPDH in

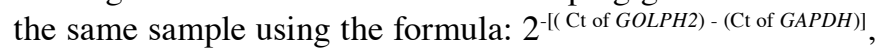
where $\mathrm{C}_{t}$ is the threshold cycle for each transcript, and then expressed as a fold-increase compared to the matched normal tissues from the same patient.

Western blotting. The fresh tissues were ground to a powder in liquid nitrogen, lysed with sampling buffer $[62.5 \mathrm{mmol} / \mathrm{l}$ Tris- $\mathrm{HCl}$ (pH 6.8),2\% SDS, $10 \%$ glycerol and 5\% 2- $\beta$-mercaptoethanol] and the protein concentrations were determined using the Bradford
Table I. Clinicopathological characteristics of patient samples and expression of GOLPH2 in gastric cancer.

Characteristics

No. of cases $(\%)$

Gender
Male
Female
Age (years)
$\geq 53$
$<53$

$185(49.9)$

$200(50.1)$

$191(49.6)$

$194(50.4)$

Clinical stage

I+II

$141(36.6)$

$\mathrm{III}+\mathrm{IV}$

$244(63.4)$

T classification

$\mathrm{T} 1+\mathrm{T} 2$

$101(26.2)$

$\mathrm{T} 3+\mathrm{T} 4$

$284(73.8)$

Lymph node metastasis

$\mathrm{N} 0+\mathrm{N} 1$

$154(40.0)$

$\mathrm{N} 2+\mathrm{N} 3$

$231(60.0)$

Metastasis

M0

$266(69.1)$

M1

119 (30.9)

Pathologic differentiation

Well or moderate

$132(34.3)$

Poor or no

Venous invasion

No

$150(61.1)$

Yes

Vital status (at follow-up)

Alive

99 (25.7)

Dead (all gastric cancer-related)

$286(74.3)$

Expression of GOLPH2

Low

$159(41.3)$

High

$226(58.7)$

GOLPH2, Golgi phosphoprotein 2.

assay (Bio-Rad Laboratories, Hercules, CA, USA). Equal amounts of protein were electrophoretically separated on $9 \%$ polyacrylamide SDS gels (SDS-PAGE) and transferred to polyvinylidene fluoride membranes (Amersham Pharmacia Biotech, Pittsburgh, PA, USA). The membranes were incubated with an anti-GOLPH2 rabbit antibody (1:1,000; Abcam, Cambridge, UK), followed by a horseradish peroxidase-conjugated anti-rabbit IgG antibody (1:2,000; Amersham Pharmacia Biotech) and the bands were detected using an enhanced chemiluminescence kit (Amersham Pharmacia Biotech) according to the manufacturer's instructions. The membranes were subsequently stripped and re-probed with an anti- $\alpha$-tubulin mouse monoclonal antibody (1:2,000; Sigma, St. Louis, MO, USA) as a loading control.

Immunohistochemistry. Immunohistochemical analysis was performed to evaluate the GOLPH2 protein expression 
in the 10 human normal gastric tissues and 385 human GC tissues. In brief, paraffin-embedded specimens were cut into $4 \mu \mathrm{m}$ sections, baked at $60^{\circ} \mathrm{C}$ for $2 \mathrm{~h}$, deparaffinized with xylene, rehydrated, subjected to antigen retrieval by microwaving in EDTA antigen retrieval buffer and treated with $3 \%$ hydrogen peroxide in methanol to quench endogenous peroxidase activity, followed by incubation with $1 \%$ bovine serum albumin to block non-specific binding. The sections were incubated with rabbit anti-GOLPH2 (1:800; Abcam) overnight at $4^{\circ} \mathrm{C}$. Normal goat serum was used as a negative control. After washing, the tissue sections were incubated with biotinylated anti-rabbit secondary antibody (Zymed, San Francisco, CA, USA) followed by streptavidin-horseradish peroxidase complex (Zymed). The sites of immunoreactivity were visualized using 3.3'-diaminobenzidine (ZSGB-BIO, Beijing, China) and the sections were counterstained with $10 \%$ Mayer's hematoxylin, dehydrated and mounted.

Two observers reviewed and independently scored the degree of immunostaining in each slide, based on the proportion of positively stained tumor cells and the intensity of staining. The proportion of positively stained tumor cells was scored as: 0 (no positive tumor cells), 1 ( $<10 \%$ positive tumor cells), 2 (10-35\% positive tumor cells), 3 (35-70\% positive tumor cells) and 4 (>70\% positive tumor cells). The intensity of immunostaining was scored using the following criteria: 0 (no staining), 1 (weak staining - light yellow), 2 (moderate staining - yellow brown) and 3 (strong staining - brown). The staining index (SI) was calculated as scores for the proportion of positive tumor cells and the staining intensity score (possible scores $=0,1,2,3,4,6,9$ or 12). An optimal cut-off value for high and low expression of GOLPH2, with respect to overall patient survival, was selected on the basis of a measure of heterogeneity using the log-rank test, where an SI score $\geq 6$ was defined as high GOLPH2 expression and an SI score of $\leq 4$ was defined as low GOLPH2 expression.

Statistical analysis. The Chi-square test was used to analyze the relationship between GOLPH2 expression and the clinicopathological characteristics of the GC patients. Bivariate correlations between study variables were calculated by Spearman's rank correlation coefficients. The end-point of this study was overall survival (OS). OS was calculated as the time from surgery until the patient succumbed to GC. Patients who were alive at the last follow-up were censored for the OS analysis. OS was calculated using the Kaplan-Meier method and compared using the log-rank test. The Cox proportional hazard models were used for univariate and multivariate analyses to determine the relationship of the clinicopathological characteristics and GOLPH2 expression with OS. Statistical analyses were carried out using SPSS 16.0 statistical software (SPSS, Chicago, IL, USA). The results were considered significant when the two-sided P-values were $<0.05$.

\section{Results}

GOLPH2 is upregulated in GC. To investigate whether GOLPH2 is upregulated in human GC, a comparative analysis was conducted to examine the expression of GOLPH2 in four pairs of primary GC tissues and the adjacent non-cancerous tissues from the same patients. qPCR analysis revealed that
A

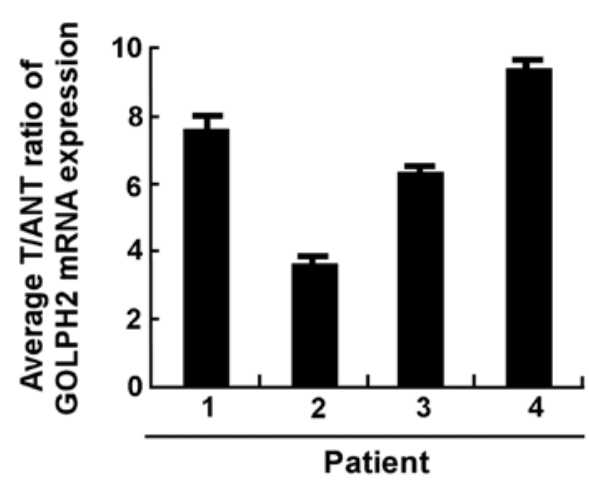

B

Patient 1 Patient 2 Patient 3 Patient 4 $\overline{\text { ANT } T} \overline{\text { ANT } T} \overline{\text { ANT T }} \overline{\text { ANT T }}$

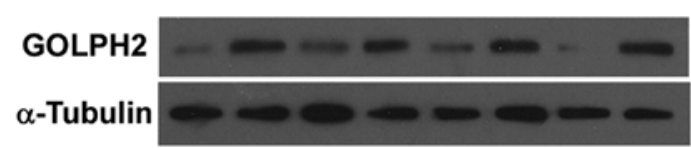

C

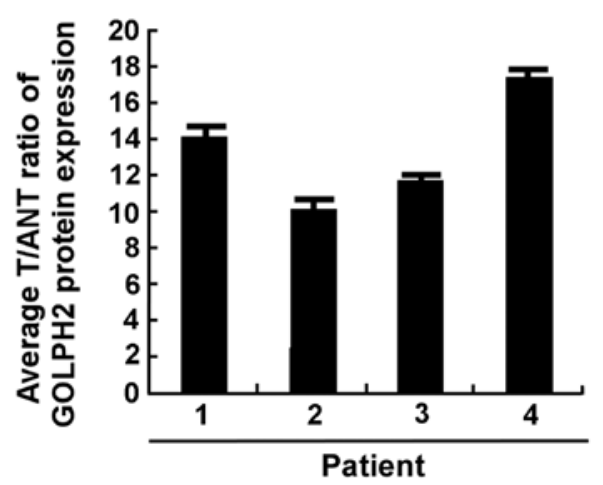

Figure 1. GOLPH2 expression is elevated in the four human primary gastric cancer tissues compared with the adjacent non-cancerous tissues. (A) Expression of GOLPH2 mRNA in primary gastric cancer tissues (T), expressed as the fold-change relative to the matched adjacent non-cancerous tissues (ANT) from the same patients, as determined by qPCR. (B) GOLPH2 expression protein in primary gastric cancer tissues $(\mathrm{T})$ and the matched adjacent non-cancerous tissues (ANT) from the same patients, as determined by western blotting. (C) Quantification of SPHK1 protein in each of the primary GC tissues (T) and GC-adjacent non-cancerous tissues (ANT) in the same patient, as determined by western blotting. GOLPH2, Golgi phosphoprotein 2.

GOLPH2 mRNA was overexpressed in the four primary GC samples. Compared to the paired adjacent non-cancerous tissues, the expression of GOLPH2 mRNA was up to 4-fold higher in the primary GC tissues (Fig. 1A). The western blot analysis demonstrated that GOLPH2 protein expression was also upregulated in all four of the human primary GC tissue samples, compared to the matched adjacent non-cancerous tissues (Fig. 1B). Notably, the protein quantification of the western blot analysis (intensity) showed that all four tumors had a $>10$-fold increase in GOLPH 2 protein compared with the tissues adjacent to the tumors (Fig. 1C). In agreement with the result of the western blot analysis, the immunohistochemical analysis also showed GOLPH2 overexpression in all four tumors in comparison with the paired non-cancerous adjacent tissues (Fig. 2). These results showed that GOLPH2 is upregulated at the mRNA and protein levels in GC. 


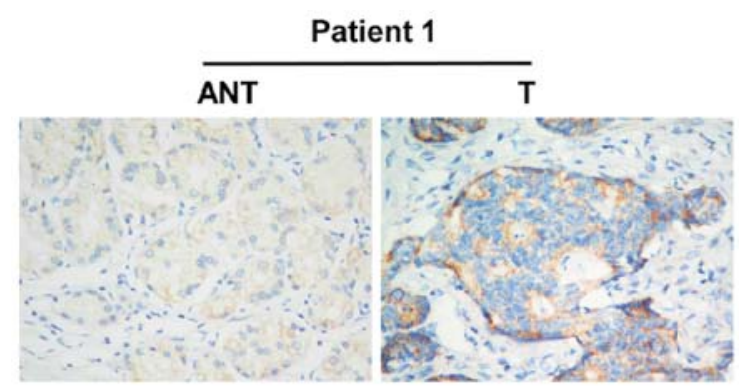

Patient 3

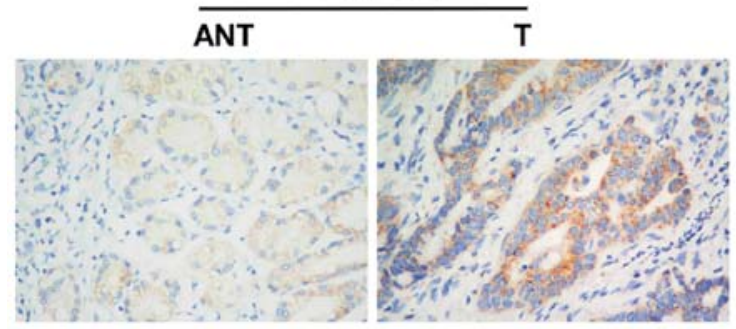

Patient 2

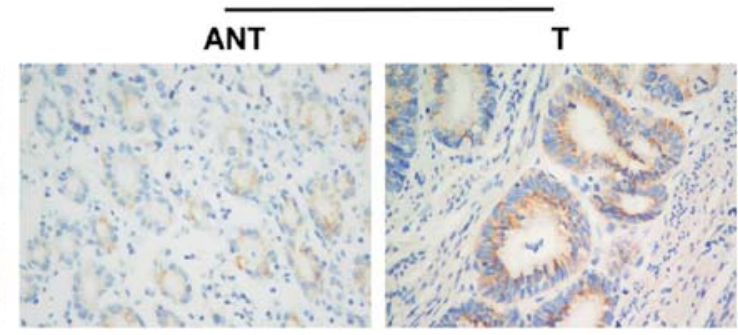

Patient 4

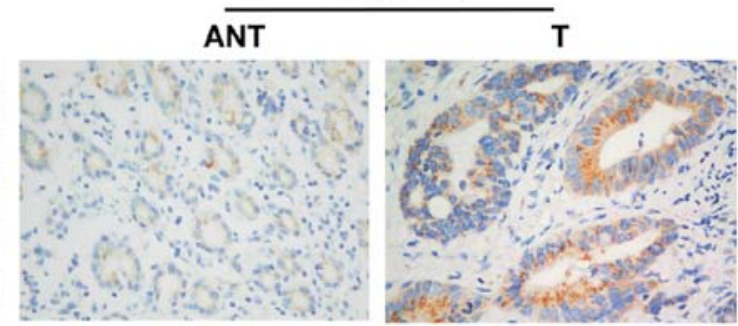

Figure 2. GOLPH2 protein expression in the four primary gastric cancer tissues (T) and the matched adjacent non-cancerous tissues (ANT) from the same patient, as determined by immunohistochemistry (IHC). GOLPH2, Golgi phosphoprotein 2.
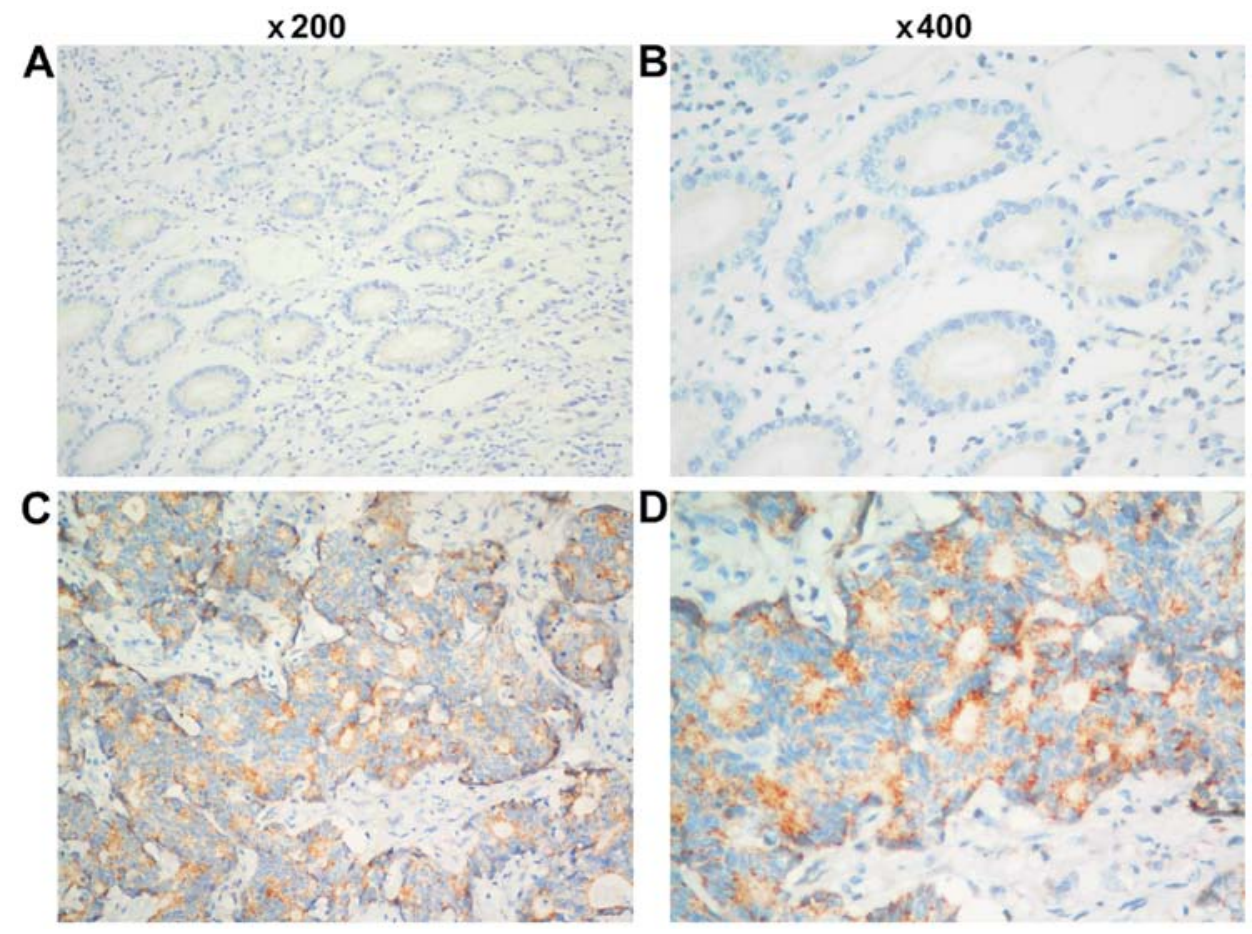

Figure 3. GOLPH2 protein is overexpressed in gastric cancer. Representative images of immunohistochemical (IHC) analysis of GOLPH2 in normal human gastric tissues and 385 archived, primary gastric cancer specimens. (A and B) GOLPH2 expression was only marginally detectable in normal epithelial cells [magnification, x200 for (A) and x400 for (B)]. (C and D) GOLPH2 was expressed at high levels in the primary gastric cancer lesions [magnification, x200 for (C) and x400 for (D)]. GOLPH2, Golgi phosphoprotein 2.

To confirm whether GOLPH2 is upregulated in GC, IHC analysis was performed to examine the protein expression levels of GOLPH2 in 10 paraffin-embedded normal gastric tissue samples and 385 paraffin-embedded, archived GC tissue samples. As shown in Fig. 3, GOLPH2 was overexpressed in the gastric tumors (Fig. 3C and D). By contrast, GOLPH2 was not detectable or only marginally detectable in the adjacent non-cancerous tissues from the same patients and the control, i.e., normal gastric tissues from individuals without GC (Fig. 3A and B). Strong cytoplasmic GOLPH2 staining was detected in $226(58.7 \%)$ of the tumors and weak or negative GOLPH2 cytoplasmic staining was detected in $159(41.3 \%)$ of the tumors. GOLPH2 was mainly localized in the cytoplasm of the primary GC cells, which is consistent 
Table II. Correlation between GOLPH2 expression and clinicopathological factors of gastric cancer patients.

\begin{tabular}{|c|c|c|c|}
\hline \multirow[b]{2}{*}{ Characteristics } & \multicolumn{2}{|c|}{ GOLPH2 } & \multirow[b]{2}{*}{ P-value } \\
\hline & Low or none no. of cases $(\%)$ & High no. of cases (\%) & \\
\hline \multicolumn{4}{|l|}{ Gender } \\
\hline Female & $85(53.5)$ & $100(47.5)$ & \multirow[t]{2}{*}{0.244} \\
\hline Male & $74(46.5)$ & $126(52.5)$ & \\
\hline \multicolumn{4}{|l|}{ Age (years) } \\
\hline$<53$ & 79 (49.7) & $112(49.6)$ & \multirow[t]{2}{*}{0.980} \\
\hline$\geq 53$ & $80(50.3)$ & $114(50.4)$ & \\
\hline \multicolumn{4}{|l|}{ Clinical stage } \\
\hline $\mathrm{I}+\mathrm{II}$ & $73(45.9)$ & $68(30.1)$ & \multirow[t]{2}{*}{0.002} \\
\hline $\mathrm{III}+\mathrm{IV}$ & $86(54.1)$ & $158(69.9)$ & \\
\hline \multicolumn{4}{|l|}{$\mathrm{T}$ classification } \\
\hline $\mathrm{T} 1+\mathrm{T} 2$ & $67(42.1)$ & $34(15.0)$ & \multirow[t]{2}{*}{$<0.001$} \\
\hline $\mathrm{T} 3+\mathrm{T} 4$ & $92(57.9)$ & $192(85.0)$ & \\
\hline \multicolumn{4}{|c|}{ Lymph node metastasis } \\
\hline $\mathrm{N} 0+\mathrm{N} 1$ & $106(66.7)$ & $48(21.2)$ & \multirow[t]{2}{*}{$<0.001$} \\
\hline $\mathrm{N} 2+\mathrm{N} 3$ & $53(33.3)$ & $178(78.8)$ & \\
\hline \multicolumn{4}{|l|}{ Metastasis } \\
\hline M0 & $120(75.5)$ & $146(64.6)$ & \multirow[t]{2}{*}{0.023} \\
\hline M1 & $39(24.5)$ & $80(35.4)$ & \\
\hline \multicolumn{4}{|c|}{ Pathologic differentiation } \\
\hline Well or moderate & $44(27.2)$ & 88 (38.9) & \multirow[t]{2}{*}{0.070} \\
\hline Poor or no & $115(72.3)$ & $138(61.1)$ & \\
\hline \multicolumn{4}{|l|}{ Venous invasion } \\
\hline No & $80(50.3)$ & $70(31.0)$ & \multirow[t]{2}{*}{$<0.001$} \\
\hline Yes & $79(49.7)$ & $156(69.0)$ & \\
\hline
\end{tabular}

GOLPH2, Golgi phosphoprotein 2.

with previous studies (10-14) on the expression of GOLPH2 in other types of cancer. Taken together, these observations demonstrate that GOLPH2 is overexpressed in GC samples.

Increased expression of GOLPH2 correlates with the clinicopathological characteristics of GC. To investigate whether GOLPH2 is associated with clinical progression, the results of the IHC analysis were subjected to statistical analysis to determine the relationship of GOLPH2 with the clinicopathological characteristics of GC patients. As shown in Table II, a high GOLPH2 expression was found to be significantly correlated with the clinical stage $(\mathrm{P}=0.002)$, $\mathrm{T}$ classification $(\mathrm{P}<0.001)$, lymph node metastasis $(\mathrm{P}<0.001)$, metastasis $(\mathrm{P}=0.023)$ and venous invasion $(\mathrm{P}<0.001)$ of patients with $\mathrm{GC}$, suggesting that advanced clinical stage and histological type correlated with higher levels of GOLPH2 expression. However, the expression of GOLPH2 was not associated with gender $(\mathrm{P}=0.244)$, age $(\mathrm{P}=0.980)$ or pathological differentiation $(\mathrm{P}=0.070)$. As shown in Table III, Spearman correlations of GOLPH2 expression levels to clinical stage, $\mathrm{T}$ classification, lymph node metastasis, metastasis and venous invasion were $0.187(\mathrm{P}=0.001)$, $0.199(\mathrm{P}=0.025), 0.168(\mathrm{P}=0.024), 0.164(\mathrm{P}=0.013)$ and 0.155
Table III. Spearman correlation analysis between GOLPH2 and clinicopathological characteristics.

\begin{tabular}{|c|c|c|}
\hline \multirow[b]{2}{*}{ Variables } & \multicolumn{2}{|c|}{ GOLPH 2 expression level } \\
\hline & Spearman correlation & P-value \\
\hline Gender & 0.045 & 0.231 \\
\hline Age (years) & 0.021 & 0.316 \\
\hline Clinical stage & 0.187 & 0.001 \\
\hline $\mathrm{T}$ classification & 0.199 & 0.025 \\
\hline Lymph node metastasis & 0.168 & 0.024 \\
\hline Metastasis & 0.164 & 0.013 \\
\hline Pathologic differentiation & 0.099 & 0.089 \\
\hline Venous invasion & 0.155 & 0.030 \\
\hline
\end{tabular}

GOLPH2, Golgi phosphoprotein 2.

$(\mathrm{P}=0.030)$, respectively. Taken together, these results indicate that overexpression of GOLPH2 correlated with the clinicopathological characteristics of GC. 
Table IV. Univariate and multivariate analyses of various prognostic parameters in patients with gastric cancer Cox-regression analysis.

\begin{tabular}{|c|c|c|c|c|}
\hline \multirow{2}{*}{$\begin{array}{l}\text { Clinicopathological } \\
\text { characteristics }\end{array}$} & \multicolumn{2}{|c|}{ Univariate analysis } & \multicolumn{2}{|c|}{ Multivariate analysis } \\
\hline & HR $(95 \% \mathrm{CI})$ & P-value & HR $(95 \% \mathrm{CI})$ & P-value \\
\hline \multicolumn{5}{|l|}{ Clinical stage } \\
\hline $\mathrm{I}+\mathrm{II}$ & 1 & 0.042 & 1 & 0.048 \\
\hline III+IV & $1.220(0.789-1.691)$ & & $1.561(0.921-2.392)$ & \\
\hline \multicolumn{5}{|l|}{ T classification } \\
\hline $\mathrm{T} 1+\mathrm{T} 2$ & 1 & $<0.001$ & 1 & $<0.001$ \\
\hline $\mathrm{T} 3+\mathrm{T} 4$ & $1.793(1.327-2.259)$ & & $1.754(1.382-2.176)$ & \\
\hline \multicolumn{5}{|c|}{ Lymph node metastasis } \\
\hline $\mathrm{N} 0+\mathrm{N} 1$ & 1 & 0.001 & 1 & 0.041 \\
\hline $\mathrm{N} 2+\mathrm{N} 3$ & $1.402(1.049-1.810)$ & & $1.471(1.259-1.720)$ & \\
\hline \multicolumn{5}{|l|}{ Metastasis } \\
\hline M0 & 1 & 0.006 & 1 & $<0.001$ \\
\hline M1 & $1.690(1.112-3.141)$ & & $1.695(1.282-2.241)$ & \\
\hline \multicolumn{5}{|c|}{ Pathologic differentiation } \\
\hline Well or moderate & 1 & 0.432 & 1 & 0.212 \\
\hline Poor or no & $1.121(0.843-1.491)$ & & $1.091(0.213-1.511)$ & \\
\hline \multicolumn{5}{|l|}{ Venous invasion } \\
\hline No & 1 & 0.030 & 1 & 0.062 \\
\hline Yes & $1.339(0.743-2.413)$ & & $1.531(0.713-2.011)$ & \\
\hline \multicolumn{5}{|l|}{ GOLPH2 expression } \\
\hline Low & 1 & $<0.001$ & 1 & $<0.001$ \\
\hline High & $1.843(1.117-2.651)$ & & $2.168(1.572-2.991)$ & \\
\hline
\end{tabular}

HR, hazard ratio; 95\% CI, 95\% confidence interval; GOLPH2, Golgi phosphoprotein 2.

Association of GOLPH2 with overall survival in GC. Univariate analysis using a Cox proportional hazards model was used to investigate the relationship of the clinicopathological characteristics of patients with OS (Table IV). Overexpression of GOLPH2 was associated with a significantly increased risk of cancer-related death $(\mathrm{P}<0.001)$ in GC patients. The HRs indicated that clinical stage $(\mathrm{P}=0.042)$, $\mathrm{T}$ classification $(\mathrm{P}<0.001)$, lymph node metastasis $(\mathrm{P}=0.001)$, metastasis $(\mathrm{P}=0.006)$ and venous invasion $(\mathrm{P}=0.030)$ were correlated with a shorter OS.

The Kaplan-Meier analysis and the log-rank test were used to evaluate the influence of GOLPH2 expression and clinicopathological factors on survival in more details. As shown in Fig. 4, the duration of OS was significantly different in patients with low and high GOLPH2 expression. The high GOLPH2 expression group had a shorter median OS than the low GOLPH2 expression group (25 months vs. 34 months). The cumulative 5-year OS rate was $39.1 \%$ [95\% confidence interval (CI), 0.254-0.514] in the low GOLPH2 expression group, compared to only $19.5 \%$ (95\% CI, 0.152-0.273) in the high GOLPH2 expression group (Fig. 4A, P<0.001).

Moreover, we evaluated the prognostic value of GOLPH2 expression in selected patient subgroups. A significant difference was observed in the OS times of patients between the low and high GOLPH2 expression groups in the clinical stages I-II subgroup ( $\mathrm{P}<0.001$; Fig. 4B). Additionally, the patients in the clinical stages III-IV subgroup ( $\mathrm{P}<0.001$; Fig. 4C) with tumors exhibiting a high GOLPH2 expression had significantly lower OS rates compared with those with a low level of GOLPH2 expression. Furthermore, it is noteworthy that the expression of GOLPH2 was markedly correlated with the survival of GC patients, even after the patients were stratified by their clinicopathologic classification (Fig. 5A-F). In the T classification, the differences of survival curves according to GOLPH2 expression were observed in $\mathrm{T} 1+\mathrm{T} 2$ classification panel $(\mathrm{P}<0.001)$ and $\mathrm{T} 3+\mathrm{T} 4$ classification panel $(\mathrm{P}=0.049)$. In the $\mathrm{N} 0+\mathrm{N} 1$ classification panel of the lymph node metastasis, patients with a low GOLPH2 expression exhibited an improved OS $(\mathrm{P}<0.001)$. In the $\mathrm{N} 2+\mathrm{N} 3$ classification panel, patient OS was also significantly different between the two groups $(\mathrm{P}=0.048)$. In the metastasis, the differences of survival curves according to GOLPH2 expression were observed in the M0 classification panel $(\mathrm{P}<0.001)$ and $\mathrm{M} 1$ classification panel $(\mathrm{P}=0.047)$.

Expression of GOLPH2 is associated with prognosis in GC. Multivariate analysis was performed using the complete clinicopathological data for all 385 GC patients (Table IV). The variables considered in the analysis were clinical stage, $\mathrm{T}$ classification, lymph node metastasis, metastasis, pathologic 

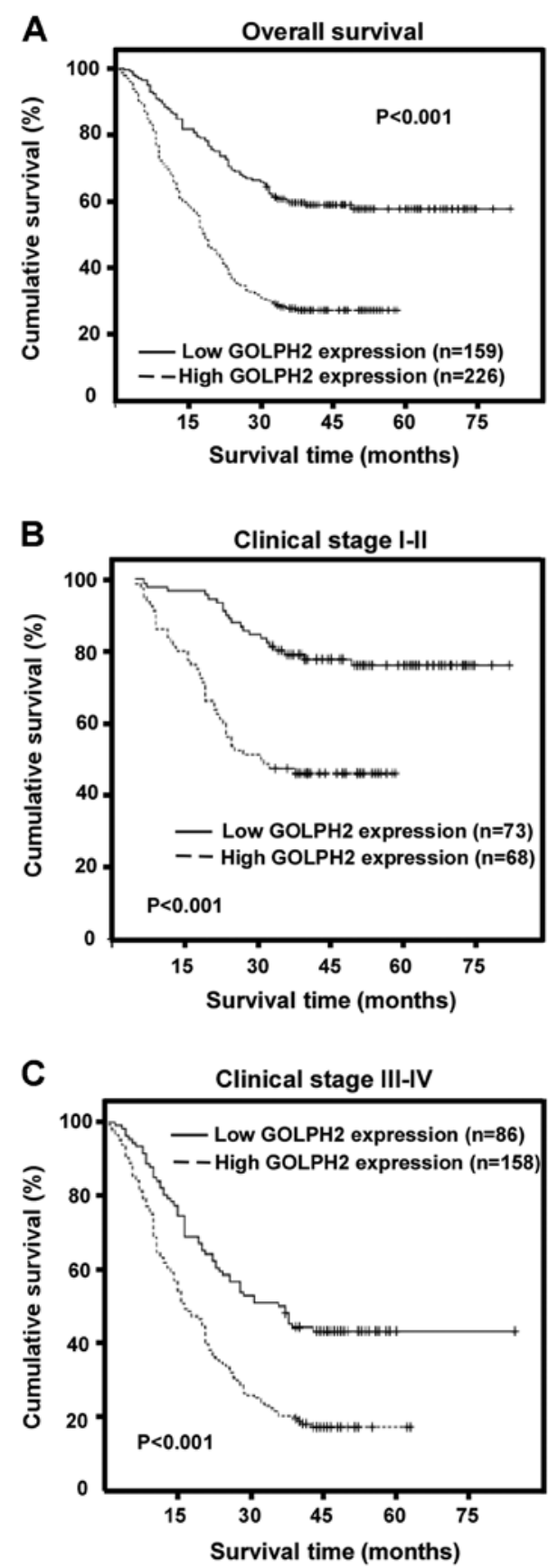

Figure 4. Kaplan-Meier survival analysis (log-rank) for 385 gastric cancer patients with a low versus a high GOLPH2 expression tumors. (A) Overall survival $(\mathrm{P}<0.001)$. (B) Clinical stage I-II. (C) Clinical stage III-IV. GOLPH2, Golgi phosphoprotein 2.

differentiation, venous invasion and GOLPH2 expression. Only clinical stage $(\mathrm{HR}=1.561,95 \% \mathrm{CI}, 0.921-2.392, \mathrm{P}=0.048), \mathrm{T}$ classification $(\mathrm{HR}=1.754,95 \% \mathrm{CI}, 1.382-2.176, \mathrm{P}<0.001)$, lymph node metastasis $(\mathrm{HR}=1.471,95 \% \mathrm{CI}, 1.259-1.720, \mathrm{P}=0.041)$, metastasis $(\mathrm{HR}=1.695,95 \% \mathrm{CI}, 1.282-2.241, \mathrm{P}<0.001)$ and GOLPH2 expression $(\mathrm{HR}=2.168,95 \%$ CI, 1.572-2.991, $\mathrm{P}<0.001)$ were independent prognostic factors of poor OS.

\section{Discussion}

To the best of our knowledge, this is the first systematic study of GOLPH2 expression in GC. The key findings of this study suggest for the first time, that the expression of GOLPH2 significantly correlates with prognosis and survival in GC patients. We found that GOLPH2 mRNA and protein levels were upregulated in human GC tissues and GOLPH2 expression was significantly associated with the clinicopathological characteristics of GC, as well as a significantly shorter OS. Moreover, GOLPH2 was a poor prognostic factor in the univariate analysis and an independent prognostic indicator in the multivariate analysis. Taken together, our study suggests that GOLPH2 is a novel predictive marker for clinical outcome in GC.

Firstly, we examined the expression of GOLPH2 in paired primary GC tissues and the adjacent non-cancerous tissues and also a large cohort of paraffin-embedded GC tissues. The results showed that the upregulation of GOLPH 2 mRNA and protein expression occurs frequently in human $\mathrm{GC}$, suggesting that the overexpression of GOLPH2 may be associated with the development and progression of GC. Notably, positive GOLPH2 staining was specifically detected in the tumor cells in the 385 paraffin-embedded archived GC specimens, whereas the adjacent non-cancerous tissues and normal gastric tissues from patients without GC expressed little, if any, GOLPH2. GOLPH2, a resident Golgi type II transmembrane protein, was originally found to be strongly overexpressed in hepatocytes from patients with viral and non-viral liver disease, including hepatitis, cirrhosis, HCC and non-liver malignancies (9, 15-22). However, the high level of sGOLPH2 had no correlation with overall patient survival or the clinicopathological characteristics of HCC, including tumor size, tumor type, Child-Pugh classification or TNM classification (23). GOLPH2 mRNA and protein expression are also upregulated in prostate cancer (11). GOLPH2 potentially serves as a diagnostic tool in prostate cancer and was predictive of poor prognosis, as indicated by the univariate and multivariate analyses $(11,24)$. However, GOLPH2 was not associated with tumor stage, differentiation grade, preoperative prostate-specific antigen (PSA) levels or disease-free survival in prostate cancer (11). In seminoma, the GOLPH2 protein may serve as a novel immunohistochemical marker. However, GOLPH2 had no correlation with the clinicopathological characteristics of seminoma patients (13). In renal cell cancer, GOLPH2 was only associated with the histological type, but had no direct diagnostic or prognostic value (12). GOLPH2 was also upregulated in lung adenocarcinoma tissues and lung adenocarcinoma cell lines; however, the upregulation of GOLPH2 did not correspond to detectable levels of GOLPH2 in serum (14). Collectively, these findings indicate that GOLPH2 may play an important role in the development and progression of various types of cancer, which led us to investigate whether GOLPH2 is also a novel marker for progression and OS in GC.

The statistical analysis revealed a significant correlation between GOLPH2 expression and clinical stage, T classification, lymph node metastasis, metastasis and venous invasion in $\mathrm{GC}$, further supporting the hypothesis that GOLPH2 plays a role in the progression of GC. Moreover, a high expression of GOLPH2 was a predictor of poor prognosis in GC patients in the univariate and multivariate analyses. Notably, there was a significant correlation between shorter OS and a high GOLPH2 expression, suggesting that GOLPH2 is a useful prognostic marker of OS in GC patients. Of note is the signifi- 

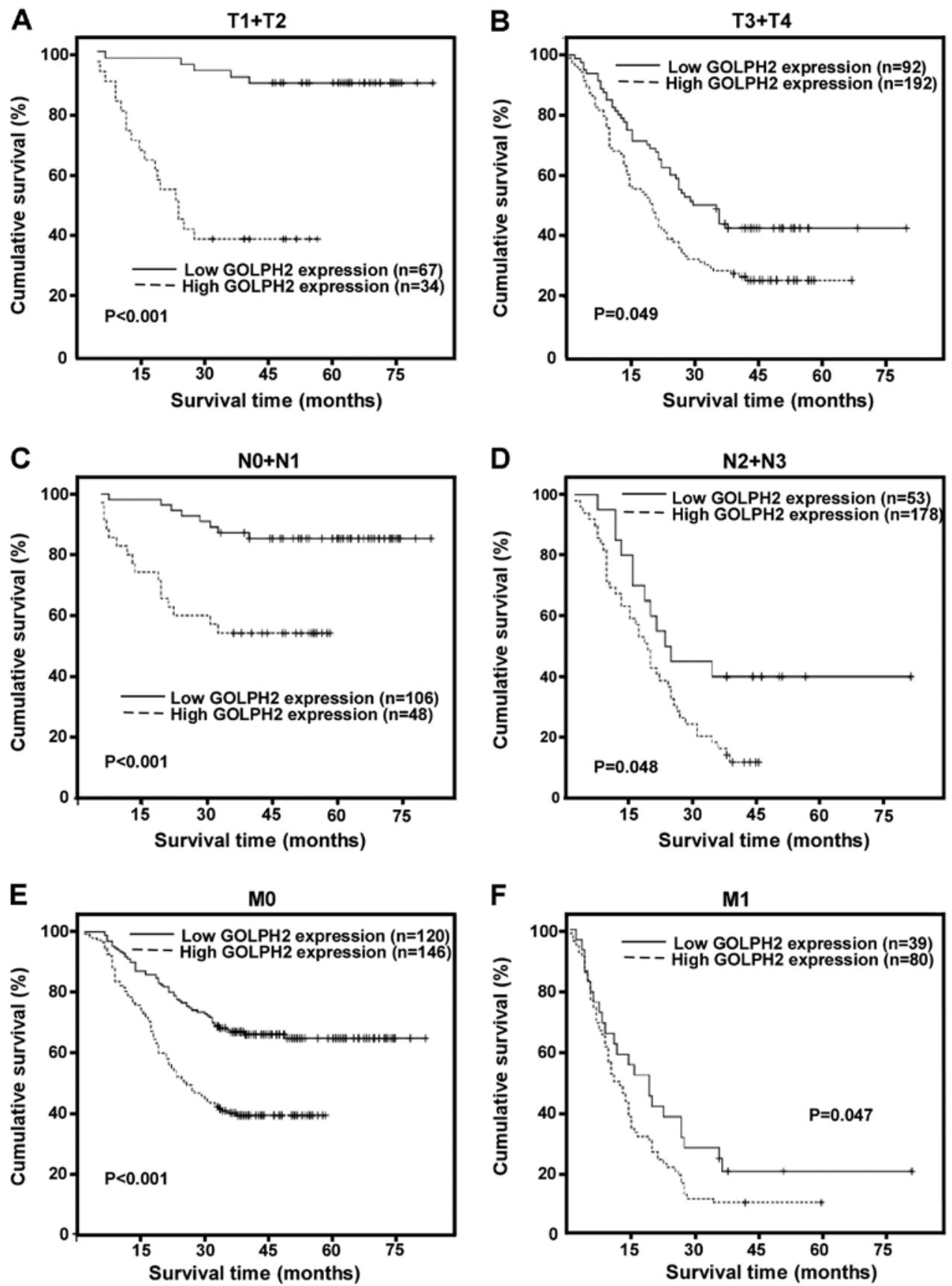

Figure 5. Overall survival curves stratified by GOLPH2 levels according to T, N, M classifications. Statistical analysis of the difference between tumors with a high and low GOLPH2 expression in the (A) T1+T2, (B) T3+T4, (C) N0+N1, (D) N2+N3, (E) M0 and (F) M1 patient subgroups. GOLPH2, Golgi phosphoprotein 2.

cant correlation between shorter overall survival times of patients and a high GOLPH2 expression in the early (clinical stage I-II) and late (clinical stage III-IV) stage subgroups, even after the patients were stratified by their clinicopathologic classification, suggesting that GOLPH2 is a useful prognostic marker for all GC patients.

In summary, we have demonstrated that an elevated expression of GOLPH2 is significantly associated with the development and progression of GC. GOLPH2 has potential as a novel and independent biomarker of prognosis in GC patients. Evidence from a multicentre study suggested that GOLPH2 functions as a serum maker for the early detection and recurrence of HCC. Compared with $\alpha$-fetoprotein (AFP), sGOLPH2 had a much higher sensitivity and specificity for the detection of early stage of HCC (10). In addition to serum or surgical tissues, whether such an important biomarker can be detected in the gastric fluid of GC patients remains to be determined. Analysis of such samples would significantly contribute to the detection of GC at the earliest possible stage and may indicate the most appropriate and effective treatment for GC patients, thereby improving their chances of recovery and survival.

\section{Acknowledgements}

The Science and Technology Department of Guangdong Province, China, (no. 1111252000047); Guangzhou Municipal 
University Science and Technology Projects (no. 08A040), Guangzhou Medical and Health Science and Technology Projects (no. 201102A213119).

\section{References}

1. Jemal A, Bray F, Center MM, Ferlay J, Ward E and Forman D: Global cancer statistics. CA Cancer J Clin 61: 69-90, 2011.

2. Roder DM: The epidemiology of gastric cancer. Gastric Cancer 5 (Suppl 1): 5-11, 2002.

3. Paoletti X, Oba K, Burzykowski T, et al: Benefit of adjuvant chemotherapy for resectable gastric cancer: a meta-analysis. JAMA 303: 1729-1737, 2010.

4. Liu JH, Song LB, Zhang X, et al: Bmi-1 expression predicts prognosis for patients with gastric carcinoma. J Surg Oncol 97 267-272, 2008

5. Gou HF, Chen XC, Zhu J, et al: Expressions of COX-2 and VEGF-C in gastric cancer: correlations with lymphangiogenesis and prognostic implications. J Exp Clin Cancer Res 30: 14, 2011.

6. Aoki M, Ishigami S, Uenosono Y, et al: Expression of BMP-7 in human gastric cancer and its clinical significance. $\mathrm{Br} \mathrm{J}$ Cancer 104: 714-718, 2011.

7. Zhou F, Li N, Jiang W, et al: Prognosis significance of HER-2/neu overexpression/amplification in Chinese patients with curatively resected gastric cancer after the ToGA clinical trial. World J Surg Oncol 10: 274, 2012.

8. Li W, Yu CP, Xia JT, et al: Sphingosine kinase 1 is associated with gastric cancer progression and poor survival of patients. Clin Cancer Res 15: 1393-1399, 2009.

9. Kladney RD, Bulla GA, GuoL, et al: GP73, a novel Golgi-localized protein upregulated by viral infection. Gene 249: 53-65, 2000.

10. Mao Y, Yang H, Xu H, et al: Golgi protein 73 (GOLPH2) is a valuable serum marker for hepatocellular carcinoma. Gut 59: $1687-1693,2010$.

11. Kristiansen G, Fritzsche FR, Wassermann K, et al: GOLPH2 protein expression as a novel tissue biomarker for prostate cancer: implications for tissue-based diagnostics. Br J Cancer 99 : 939-948, 2008.
12. Fritzsche FR, Riener MO, Dietel M, Moch H, Jung K and Kristiansen G: GOLPH2 expression in renal cell cancer. BMC Urol 8: 15, 2008.

13. Fritzsche FR, Kristiansen G, Riener MO, Dietel M and Oelrich B: GOLPH2 expression may serve as diagnostic marker in seminomas. BMC Urol 10: 4, 2010.

14. Zhang F, Gu Y, Li X, Wang W, He J and Peng T: Up-regulated Golgi phosphoprotein 2 (GOLPH2) expression in lung adenocarcinoma tissue. Clin Biochem 43: 983-991, 2010.

15. Marrero JA, Romano PR, Nikolaeva O, et al: GP73, a resident Golgi glycoprotein, is a novel serum marker for hepatocellular carcinoma. J Hepatol 43: 1007-1012, 2005.

16. Riener MO, Stenner F, Liewen H, et al: Golgi phosphoprotein 2 (GOLPH2) expression in liver tumors and its value as a serum marker in hepatocellular carcinomas. Hepatology 49: 1602-1609, 2009.

17. Kladney RD, Cui X, Bulla GA, Brunt EM and Fimmel CJ: Expression of GP73, a resident Golgi membrane protein, in viral and nonviral liver disease. Hepatology 35: 1431-1440, 2002.

18. Hu JS, Wu DW, Liang S and Miao XY: GP73, a resident Golgi glycoprotein, is sensibility and specificity for hepatocellular carcinoma of diagnosis in a hepatitis B-endemic Asian population. Med Oncol 27: 339-345, 2010.

19. Wang M, Long RE, Comunale MA, et al: Novel fucosylated biomarkers for the early detection of hepatocellular carcinoma. Cancer Epidemiol Biomarkers Prev 18: 1914-1921, 2009.

20. Liu X, Wan X, Li Z, Lin C, Zhan Y and Lu X: Golgi protein 73(GP73), a useful serum marker in liver diseases. Clin Chem Lab Med 49: 1311-1316, 2011.

21. Iftikhar R, Kladney RD, Havlioglu N, et al: Disease- and cell-specific expression of GP73 in human liver disease. Am J Gastroenterol 99: 1087-1095, 2004.

22. Hann HW, Wang M, Hafner J, et al: Analysis of GP73 in patients with HCC as a function of anti-cancer treatment. Cancer Biomark 7: 269-273, 2010.

23. Ozkan H, Erdal H, Tutkak H, et al: Diagnostic and prognostic validity of Golgi protein 73 in hepatocellular carcinoma. Digestion 83: 83-88, 2011.

24. Laxman B, Morris DS, Yu J, et al: A first-generation multiplex biomarker analysis of urine for the early detection of prostate cancer. Cancer Res 68: 645-649, 2008. 